Baldassare Pastore. Catedrático de Filosofía del Derecho en el Dipartimento di Giurisprudenza de la Università degli Studi di Ferrara. Coeditor de "Ars interpretandi. Rivista di ermeneutica giuridica". Autor de numerosas obras sobre la teoría jurídica, la teoría de la interpretación jurídica, la filosofía práctica, los Derechos Humanos y el multiculturalismo.

Contacto: baldassare.pastore@unife.it 


\title{
SOFT LAW Y LA TEORÍA DE LAS FUENTES DEL DERECHO
}

\author{
Baldassare Pastore \\ Università degli Studi di Ferrara
}

Fecha de recepción 4 de febrero de 2014; fecha de aceptación 4 de marzo de 2014. El artículo es fruto de un proyecto de investigación desarrollado en el Dipartimento di Giurisprudenza de la Università degli Studi di Ferrara.

\section{Resumen}

El soft law hace referencia a reglas de conducta que en principio no tienen fuerza jurídica vinculante, aunque produzcan efectos prácticos. El artículo investiga esta noción a la luz de la relación entre fuentes del derecho e interpretación. El soft law hace referencia a una serie de fenómenos relacionados a la positivización jurídica y a las prácticas interpretativas en el ámbito del derecho internacional, derecho de la UE y derecho nacional. El soft law desempeña distintas funciones que coexisten con el sistema de hard law. El soft law juega un papel en el ordenamiento jurídico que muestra la gradual diferenciación de su normatividad.

\section{Palabras clave}

Soft law, interpretación, fuentes del derecho, normatividad, técnicas de regulación

\section{Abstract}

Soft law concerns rules of conduct which, in principle, have no legally binding force but which nevertheless may have practical effects. The article examines this notion in the light of the relationship between legal sources and interpretation. Soft law refers to a range of phenomena related to legal positivization and interpretative practices in the field of international law, EU law and national law. Soft law performs various functions, traveling in tandem with hard law. Soft law plays a role in legal order, which shows a gradual differentiation of its normativity. 


\section{Keywords}

Soft law, interpretation, legal sources, normativity, techniques of regulation

\section{Los usos de soft law}

El sintagma soft law se utiliza para indicar una serie de actos no homogéneos en cuanto a su origen y naturaleza que, aunque aparentemente sin efectos jurídicos vinculantes, resultan ellos mismos jurídicamente relevantes en distintas maneras.

Se trata de una noción eminentemente doctrinal ${ }^{1}$ de la cual se conocen muchos ejemplos en el actual panorama jurídico, caracterizado por un enorme pluralismo y un considerable nivel de complejidad.

En el ámbito internacional, en la categoría de soft law se recogen declaraciones de principios de las Naciones Unidas, junto con resoluciones, exhortaciones, votos y apelaciones. También se incluyen normas convencionales, non-binding agreements, resoluciones, recomendaciones y declaraciones de principios de organizaciones o conferencias internacionales con valor meramente exhortatorio y no obligatorio, de orientación o programáticos, ${ }^{2}$ que encarnan nuevas exigencias de reglamentación; así como aquellas normas que, por ser parte de acuerdos aun no entrados en vigencia o finalizados, no tienen efectos jurídicos. ${ }^{3} \mathrm{Si}$ a continuación lo consideramos dentro de la estructura de las fuentes del derecho internacional, el soft law hace referencia a todos aquellos actos y procedimientos que se quedan fuera del marco indicado por el Art. 38 del Estatuto de la Corte Internacional de Justicia. La noción, por lo tanto, nos remitiría a elementos normativos sin valor de vinculación que, aunque no produzcan por sí solos algún derecho ni obligación, pueden determinar algunos efectos jurídicos y transformarse, a través del uso de los órganos competentes, en derecho inmediatamente preceptivo. ${ }^{4}$ En este sentido, el soft law juega un papel en la formación de las normas generales y, de manera

1. R. R. Baxter, “International Law in 'Her Infinite Variety”, en The International and Comparative Law Quarterly, 29, 1980, pp. 549-566; O. Elias-C. Lim, “'General Principles of Law, 'Soft' Law and the Identification of International Law”, en Netherlands Yearbook of International Law, 28, 1997, pp. 4-7, 45-49; A. Poggi, “Soft Law nell’ordinamento comunitario”, en Annuario 2005. L'integrazione dei sistemi costituzionali europeo e nazionali, Cedam, Padova, 2007, pp. 372-380.

2. Cfr. M. Distefano, "Origini e funzioni del soft law in diritto internazionale”, en Lavoro e diritto, 17, n. 1, 2003, pp. 18-26. 3. H. Hillgenberg, "A Fresh Look at Soft Law", en European Journal of International Law, 10, n. 3, 1999, pp. 499-515.

4. El soft law puede configurarse como elemento de reconocimiento de una costumbre o de un principio general, como instrumento preparatorio de un tratado internacional, como medio interpretativo o integrativo de normas. Véase M. Distefano, “Origini e funzioni del soft law in diritto internazionale”, pp. 20, 26-29. 
más general, en la elaboración de standards uniformes (por ejemplo, la defensa de los derechos humanos y la protección del medioambiente) que reflejan el interés general de la comunidad mundial dentro de su lenta y difícil evolución hacia una cooperación institucional activa. ${ }^{5}$ En este escenario, el uso del soft law representa un modo fluido y flexible por parte de los Estados, firmes tutores de sus esferas de soberanía, para hacer frente a la defensa de sus intereses particulares junto con la imposibilidad de adoptar una regulación vinculante y universal. Tal derecho parece caracterizarse como un orden de normativa graduada, ${ }^{6}$ apto para acoger las contribuciones de los distintos sujetos presentes en la escena global y las múltiples formas de presencia jurídica que estos manifiestan, respondiendo dinámicamente a una situación de constante desarrollo. ${ }^{7}$

El uso de formas de soft law juega un papel no secundario en la construcción de un derecho uniforme a nivel transnacional. Un ejemplo relevante consiste en el uso de los principios Unidroit como directrices que orientan la praxis comercial. ${ }^{8}$ Tales principios pueden considerarse como elementos para la redacción de contratos comerciales internacionales, como normas regulatorias de estas relaciones, como criterios aplicables en la resolución de eventuales controversias por parte de los jueces estatales y árbitros, como estándares para la interpretación o la integración jurídica y como fuentes de inspiración para la codificaciones nacionales e internacionales. El hecho de que los principios Unidroit no estén destinados a convertirse en un instrumento vinculante, aunque sean aceptados por los operadores del derecho y la comunidad de juristas únicamente por su capacidad de persuasión, hace que se adapten muy bien a las condiciones variables del comercio internacional y que desempeñen una importante función ordinativa.

Otro ejemplo de ductilidad jurídica es el de los Principles of European Contract Law. En este caso se trata de criterios ordenantes utilizables por los sujetos que estipulan

\footnotetext{
5. B. Pastore, "Il diritto internazionale in un mondo in trasformazione: verso un diritto giurisprudenziale?", en Ars Interpretandi, 6, 2001, pp. 158-163; F. Francioni, "International 'Soft Law': A Contemporary Assesment”, en V. Lowe, M. Fitzmaurice (eds.), Fifty Years of the International Court of Justice. Essays in Honour of Sir Robert Jennings, Cambridge University Press, Cambridge, 1996, pp. 167-178; D. Thürer, "Soft Law", en Encyclopedia of Public International Law, vol. 4, Elsevier, Amsterdam-London-New York-Oxford-Paris-Shannon-Tokio, 2000, pp. 452-460; J.J. Kirton, M.J. Trebilcock (eds.), Hard Choices, Soft Law. Voluntary Standards in Global Trade, Environment and Social Governance, Ashgate Publishing Company, Aldershot, 2004.

6. P. Weil, “Towards Relative Normativity in International Law?”, en The American Journal of International Law, 77, 1983, pp. 413-415; U. Fastenrath, "Relative Normativity in International Law", en European Journal of International Law, 4, 1993, pp. $330-332$.

7. K. W. Abbott - D. Snidal, "Hard and Soft Law in International Governance", en International Organization, 54, n. 3, 2000, pp. 421-424, 454-455; D. Shelton, "Introduction. Law, Non-Law and the Problem of Soft Law”, en D. Shelton (ed.), Commitment and Compliance. The Role of Non-Binding Norms in the International Legal System, Oxford University Press, Oxford-New York, 2003, pp. 1-18.

8. M. J. Bonell, "Soft Law and Party Autonomy: The Case of the Unidroit Principles", en Loyola Law Review, 51, 2005, pp. 229 ss.
} 
contratos, por los jueces y árbitros en decisiones sobre las controversias contractuales y por los legisladores que preparan reglas contractuales tanto a nivel europeo como a nivel nacional. Además, no hay que subestimar el objetivo - a largo plazo - que aspira a la armonización del derecho contractual dentro del ordenamiento europeo.

El soft law se usa cada vez más en la Unión Europea. En este caso también nos referimos a reglas de conducta que, en principio, no tienen una fuerza jurídicamente vinculante y que, sin embargo, pueden producir efectos prácticos. ${ }^{9}$ Un ejemplo significativo son las recomendaciones de la Comisión y del Consejo que, marcando las directrices, pueden referirse a amplias áreas de estrategia política dentro del marco de la coordinación de las relaciones entre los Estados "europeizados" y del balance entre unidad y diversidad. Además, no hay que olvidarse de los códigos de conducta, los libros verdes y libros blancos, las acciones de programa, las comunicaciones, las resoluciones, las orientaciones, las declaraciones, los consejos y las instrucciones que operan en la praxis de las instituciones comunitarias y dentro del contexto de sus interrelaciones. ${ }^{10} \mathrm{~A}$ veces se trata de indicaciones que pueden ser transformadas en hard law a través de decisiones judiciales, administrativas o legislativas. ${ }^{11}$

En líneas generales, los actos atribuibles a tal fenómeno se pueden clasificar en tres funciones: ${ }^{12}$ la de pre-law, relativa a los instrumentos preparatorios de actos jurídicos vinculantes; ${ }^{13}$ la función de post-law, de la cual forman parte los instrumentos de interpretación, implementación y actuación de actos vinculantes; ${ }^{14}$ y la función para-law que hace referencia a instrumentos alternativos a actos vinculantes. ${ }^{15}$

En cualquier caso, dentro del ordenamiento de la Unión Europea, la falta de vinculación de los actos de soft law no implica que estos no tengan efecto sobre la conducta de los Estados miembros, instituciones e individuos, aunque sea de un modo atenuado con respecto a los que habitualmente están relacionados con normas jurídicas vinculantes. Podemos destacar aquí una cuestión central para cualquier consideración sobre el papel que desempeña esta forma de regulación: es decir, la relación entre legalidad, legitimidad

9. F. Snyder, “"Soft law» e prassi istituzionale nella Comunità europea”, en Sociologia del diritto, 20, n. 1, 1993, p. 80.

10. K. C Wellens - G. M. Borchardt, "Soft Law in European Community Law", en European Law Review, 14, 1989, pp. 289, 296-302; L. Senden, Soft Law in European Community Law, Hart Publishing, Oxford and Portland (Oregon), 2004, pp. 107 ss., 118 ss., 123 ss.; A. Poggi, “Soft Law nell'ordinamento comunitario”, pp. 394-400.

11. L. Senden, Soft Law in European Community Law, pp. 119-120, 321 ss., 361 ss., 401 ss., 457-461; F. Snyder, “«Soft law» $e$ prassi istituzionale nella Comunità europea", pp. 97-101.

12. L. Senden, Soft Law in European Community Law, pp. 118-120, 122 ss.

13. Se trata, típicamente, de los libros blanco y los libros verdes.

14. Nos enfrentamos, en estos casos, con directrices, códigos de conducta, comunicaciones interpretativas.

15. Un ejemplo son las recomendaciones, los consejos, las comunicaciones no interpretativas, que se ponen como actos alternativos a la legislación. De todas formas, hay que destacar que las recomendaciones desempeñan una función pre-law y post-law. 
y eficacia ${ }^{16}$ que remite a la práctica de reconocimiento y al consenso de los sujetos implicados. ${ }^{17}$ Se perfila así una idea de derecho que no depende solo del reconocimiento formal/procedural, sino también de su capacidad de ser efectivo, es decir respetado por los ciudadanos y aplicado por los órganos institucionales.

De todos modos, en el ámbito del derecho de la UE, el uso del soft law adquiere un carácter principalmente funcional, coherentemente con los rasgos característicos de este ordenamiento, en el cual las herramientas jurídicas vehiculan primariamente las políticas adoptadas con objetivos específicos y en determinados sectores. El soft law es un instrumento de regulación entre instituciones, en ausencia de definiciones constitucionales precisas de los límites y de los ámbitos de competencia. ${ }^{18}$ Realiza una labor de unificación, armonización, coordinación y cooperación ${ }^{19}$ y se caracteriza como instrumento de governance. ${ }^{20}$

La técnica del soft law presenta algunos rasgos típicos: tendencia a regular a través de principios, con preceptos formulados con carácter persuasivo; creación de procedimientos estables para supervisar los fenómenos regulados; implicación de múltiples actores que operan en ámbitos y a niveles distintos. Otro aspecto específico del soft law europeo-comunitario es el hecho de que está sometido, de alguna manera, a un control de legalidad ejercido por el Tribunal de Justicia de la Unión Europea. ${ }^{21}$

16. Cfr. F. Snyder, “'Soft law’ e prassi istituzionale nella Comunità europea”, p. 103; U. Mörth, “Conclusion”, en Id. (ed.), Soft Law in Governance and Regulation. An Interdisciplinary Analysis, Edward Elgar, Cheltenham (UK) - Northampton (Mass., USA), 2004, pp. 196-199.

17. L. Senden, Soft Law in European Community Law, pp. 477-483; H. Frykman-U. Mörth, "Soft Law and Three Notions of Democracy: The Case of the EU”, in U. Mörth (ed.), Soft Law in Governance and Regulation. An Interdiscipinary Analysis, pp. 155-170; A. Catania, Metamorfosi del diritto. Decisione e norma nell'età globale, Laterza, Roma-Bari, 2008, pp. 13, 99 $100,148-149$.

18. Cfr. B. Caruso, "Il diritto del lavoro tra hard e soft law: nuove funzioni e nuove tecniche normative", en M. Barbera (ed.), Nuove forme di regolazione. Il metodo aperto di coordinamento delle politiche sociali, Giuffré, Milano, 2006, pp. 85-86. 19. A. Poggi, “Soft Law nell'ordinamento comunitario", pp. 393-394, 401; G. Falkner, O. Treib, M. Hartlapp, S. Leiber, Complying with Europe. EU Harmonisation and Soft Law in the Member States, Cambridge University Press, Cambridge-New York, 2005.

20. Cfr. M. Cini, “The Soft Law Approach: Commission Rule-Making in the EU's State Aid Regime”, en Journal of European Public Policy, 8, n. 2, 2001, pp. 192-207; K. Jacobsson, "Soft Regulation and the Subtle Transformation of States: the Case of EU Employment Policy", in Journal of European Social Policy, 14, 2004, pp. 355-370; F. Bano, "Diritto del lavoro e nuove tecniche di regolazione: il soft law", in Lavoro e diritto, 17, n. 1, 2003, pp. 50-52, 56, 58-59, 66; R. Manfrellotti, Sistema delle fonti e indirizzo politico nelle dinamiche dell'integrazione europea, Giappichelli, Torino, 2004 pp. 192-199; E. Pariotti, Normatività giuridica e governance delle tecnologie emergenti, en G. Guerra, A. Muratorio, E. Pariotti, M. Piccinni, D. Ruggiu (eds.), Forme di responsabilità, regolazione e nanotecnologie, Il Mulino, Bologna, 2011, pp. 524-531.

La relación entre soft law y governance se analiza desde el punto de vista jurídico, sociológico y político, en relación a la Unión Europea y a nivel de las organizaciones internacionales, en U. Mörth (ed.), Soft Law in Governance and Regulation. An Interdiscipinary Analysis. Una fuerte crítica a la difusión del soft law como instrumento de governance en área europeo-comunitaria es propuesta por A. Somma, "Soft law sed law. Diritto morbido e neocorporativismo nella costruzione dell'Europa dei mercati e nella distruzione dell'Europa dei diritti”, en Rivista critica del diritto privato, 26, n. 3, 2008, pp. 437-467.

21. F. Bano, "Diritto del lavoro e nuove tecniche di regolazione: il soft law", p. 53; B. Caruso, "Il diritto del lavoro tra hard e soft law: nuove funzioni e nuove tecniche normative", pp. 87- 89. Sobre el papel del soft law en la interpretación del 
El soft law está presente también en los ordenamientos nacionales. En este ámbito, de hecho, los fenómenos que necesitan cumplir con la necesidad de flexibilidad y articulación de la intervención regulativa son varios. Se trata de experiencias relacionadas con la difusión de organismos como autoridades administrativas independientes y comisiones éticas, designadas para establecer y hacer respectar las reglas deontológicas y comportamentales de determinadas profesiones o actividades. Estos sujetos operan principalmente a través de la formulación de consejos, directivas y recomendaciones que, aunque no produzcan efectos jurídicos y prescindan de una estricta disciplina vinculante, mantienen una relevancia en el mundo jurídico. ${ }^{22}$ Asimismo, estos fenómenos están relacionados con la definición de aquellas formas de autodeterminación normativa que necesitan de disciplinas ligeras, elásticas y flexibles como respuesta a la complejidad del orden social. ${ }^{23}$ Las "normas ligeras" se utilizan también en algunos sectores de ordenación vigente para orientar la actividad de los destinatarios hacia un objetivo, sin necesidad de imponer un determinado comportamiento. ${ }^{24}$

Estos fenómenos indican un cambio en el modelo de regulación: no tan orientado hacia normas rígidas, impuestas a los ciudadanos en general y basadas en circunstancias analíticas, sino más bien enfocado a la necesidad del proceso normativo de adaptarse a las dinámicas sociales, económicas y tecnológicas en constante mutación. Estos cambios también nos llevan a un modelo más idóneo que tenga en cuenta los diferentes intereses en juego con el fin de orientar el comportamiento de los sujetos, sin obligarle a ninguno en concreto.

De este modo, la elasticidad y la versatilidad de las herramientas jurídicas, así como los mecanismos de verificación de su actuación, permiten cumplir funciones distintas: de orientación, de información y comunicación, de socialización hacia las finalidades y los valores de las reglas y del proceso de interpretación de las mismas. ${ }^{25} \mathrm{Al}$ mismo tiempo, estas características permiten conciliar la intervención normativa con la múltiples manifestaciones de pluralismo de las sociedades actuales, impidiendo que el derecho represente un factor de rigidez.

\footnotetext{
derecho comunitario y sobre su reconocimiento por parte de la Corte de Justicia, véase L. Senden, Soft Law in European Community Law, pp. 363-373; A. Poggi, "Soft Law nell'ordinamento comunitario", pp. 380-393; I. Österdahl, "The ECJ and Soft Law: Who's Afraid of the EU Fundamental Rights Charter?”, en U. Mörth (ed.), Soft Law in Governance and Regulation. An Interdiscipinary Analysis, pp. 37-60.

22. A. Predieri, L'erompere delle autorità amministrative indipendenti, Passigli Editori, Firenze-Antella, 1997, pp. 61, 66-67. 23. S. Rodotà, Repertorio di fine secolo, Laterza, Roma-Bari, 1999, pp. 170-172; S. Rodotà, "Diritto, scienza, tecnologia: modelli e scelte di regolamentazione", en Rivista critica del diritto privato, 22, n. 3, 2004, pp. 362, 272-373.

24. F. Bano, "Diritto del lavoro e nuove tecniche di regolazione: il soft law", pp. 70-72; E. Mostacci, La soft law nel sistema delle fonti: uno studio comparato, Cedam, Padova, 2008, pp. 113-125.

25. M. R. Ferrarese, "Soft law: funzioni e definizioni", en A. Somma (ed.), Soft law e hard law nelle società postmoderne, Giappichelli, Torino, 2009, pp. 80-82.
} 
La disciplina jurídica, dentro de un contexto caracterizado por la convivencia y el concurso de varias fuentes y múltiples sujetos reguladores, intenta ofrecer respuestas diversificadas, adaptándose a las distintas dinámicas sociales. El derecho adquiere así fórmulas expresivas más fluidas.

En un panorama caracterizado por la relativización del principio de tipicidad de las formas y de las fuerzas normativas, así como por la creciente variedad de las reglas jurídicas, se encuentra, con sus muchos aspectos, el soft law, que representa uno de los factores que hoy en día permite la creación de la normativa jurídica y de su "reconceptualización" ${ }^{26}$

\section{Normativa graduada}

La heterogeneidad parece ser un rasgo peculiar del soft law, que manifiesta la complejidad de la dimensión jurídica. El derecho puede ser soft con respecto a su autoridad, su contenido y su eficacia, pero también puede serlo como expresión de una juridicidad en estado incoativo.

La combinación de una limitada capacidad de autoridad con un nivel variable de efectividad y relevancia jurídica ha llevado a suponer que el soft law se quede casi fuera de los limites de las que se consideran las "verdaderas" fuentes del derecho. La noción remitiría a "actos no-normativos de valor sub-legal”, a normas de contenido impreciso y con finalidad indefinida, a un derecho débilmente obligatorio, aunque a veces con una fuerza persuasiva la cuya juridicidad resulta ambigua y subestimada. ${ }^{27}$ Según este principio, siendo la capacidad de vincular y coercer rasgos típicos del derecho, estas reglas elásticas, que insisten en la persuasión más que en la obligatoriedad y se caracterizan por una obediencia prevalentemente voluntaria, no serían reglas jurídicas. $^{28}$ Sin embargo, semejantes reglas tienen su aplicación, convirtiéndose en eficaces, y funcionan como razones que tienen su papel en la justificación de las decisiones. ${ }^{29}$ De distintas maneras y a través del uso y del reconocimiento, árbitros, jueces, agencias

26. E. Pariotti, “«Soft law» e ordine giuridico ultra-statuale tra «rule of law» e democrazia”, en Ragion pratica, 32, 2009, pp. 88,102 .

27. J. Klabbers, “The Undesirability of Soft Law”, en Nordic Journal of International Law, 67, 1998, pp. 381-391; J. d'Aspremont, "Softness in International Law: A Self-Serving Quest for New Legal Materials", en The European Journal of International Law, 19, 2008, pp. 1075-1093.

28. S. Ferreri, “Tipologia”, en A. Pizzorusso, S. Ferreri, Le fonti del diritto italiano. 1. Le fonti scritte, con la colaboración de A. Gambaro e R. Sacco, Utet, Torino, 1998, pp. 299-301.

29. Cfr., en generale, B. Leiter, "Legal Indeterminacy", en Legal Theory, 1, 1995, pp. 481-485, 489 ss.; G. Itzcovich, Teorie e ideologie del diritto comunitario, Giappichelli, Torino, 2006, pp. 26-31, 429-430. 
administrativas, legisladores y otros actores del “juego" jurídico trasforman criterios no vinculantes en normas definidas, según la exigencias prácticas y la peculiaridad del comportamiento que hay que disciplinar. ${ }^{30}$

Tal vez, se podría hablar de exclusividad del soft law. Sin embargo, el carácter evasivo de la noción, bajo esta perspectiva, no parece una razón determinante para minimizar la función que un derecho elástico, desvinculado de la rigidez de la ley y sometido a la praxis interpretativa y aplicativa, puede desempeñar. ${ }^{31}$

En el escenario actual, el derecho tiende a marcar sus valores interactivos y comunicativos. Eso lo convierte en un producto abierto en muchos ámbitos, según una lógica de la posibilidad y de la oportunidad, más que por una vinculación o una sanción. Esta dinámica, expresión de la crisis actual del monopolio estatal (más especificadamente legislativo) de la producción jurídica, con la consiguiente incertidumbre de la jerarquía de las fuentes, se ve agravada por los procesos de la globalización. ${ }^{32}$ Tales procesos celebran el triunfo del mercado sobre la política, enseñando la cara de la arrogancia del poder político, y establecen relaciones multiformes y dialécticas entre los distintos productores del derecho (públicos y privados; estatales, supraestatales e infraestatales) y entre las componentes formales e informales. De esta manera, el derecho pierde sus connotaciones hard, propias de la tradición estatalista, que tiene como rasgos característicos el nexo exclusivo con la soberanía, la verticalidad de la estructura y una configuración de tipo normativo. ${ }^{33}$

La primacía del derecho legislativo está erosionada. ${ }^{34}$ La ley se configura cada vez menos como expresión jurídica “exhaustiva”. La crisis de la legislación afecta cada vez más a sus métodos de intervención en la sociedad, que parecen demasiado rígidos e incapaces de seguir caminos más articulados y complejos. Por lo tanto, es necesario extender el espectro de acción del concepto de derecho, librándole de la referencia obligada a una estructura típica que remite al modelo de autoridad. La fuente legislativa resulta contaminada por nuevas formas jurídicas, que prescinden de valores estrictamente preceptivos. ${ }^{35}$

\footnotetext{
30. Resulta emblemática, en relación al uso de actos sin efectos jurídicos vinculantes que operan como instrumentos interpretativos y/o integrativos de actos vinculantes, la sentencia Salvatore Grimaldi contra Fonds de maladies professionnelles del Tribunal de Justicia de la Comunidad Europea (proceso C-322/88) del 13 de diciembre del 1989, en Raccolta della Giurisprudenza della Corte, 1989, pp. 4407-4422. Véase también, en relación al derecho italiano, la sentencia del 16 de octubre del 2007 n. 21748 de la Corte di Cassazione, sezione I civile, en "Il Foro Italiano", CXXX, 2007, c. 3025 ss.

31. B. Pastore, "Soft law, gradi di normatività, teoria delle fonti", en Lavoro e diritto, 17, n. 1, 2003, pp. 9-11.

32. A. Catania, Metamorfosi del diritto, pp. 25, 34, 83, 147, 150; M. Vogliotti, Tra fatto e diritto. Oltre la modernità giuridica, Giappichelli, Torino, 2007, pp. 272-280; G. Zaccaria, La comprensione del diritto, Laterza, Roma-Bari, 2012, pp. 48-58.

33. M. R. Ferrarese, Il diritto al presente. Globalizzazione e tempo delle istituzioni, Il Mulino, Bologna, 2002, pp. 147, 193-194;

A. di Robilant, "Genealogies of Soft Law”, en The American Journal of Comparative Law, 54, 2006, pp. 499-501.

34. Cfr. G. Zaccaria, La comprensione del diritto, pp. 31-34, 40 ss.

35. M. R. Ferrarese, Il diritto al presente, pp. 72, 136-137.
} 
El sistema de las fuentes se desestructura y se aniquila. ${ }^{36}$ Las fuentes de multiplican y se diferencian; se difunden en un contexto abigarrado y mudable; tienden a evitar un orden rígido y jerárquico, piramidal, buscando coordinaciones de tipo reticular. ${ }^{37} \mathrm{~A}$ la debilidad de la capacidad regulatoria de la legislación corresponde la creciente relevancia de las herramientas jurídicas soft, suaves, "fluidas", capaces de adaptarse a contextos diferentes y de influenciar las estructuras presentes y futuras, abiertas a aportaciones y contribuciones que llegarán in itinere. ${ }^{38}$

De esta manera, en un universo jurídico repleto y heterogéneo - que muestra las características de una estructura multipolar, de tipo horizontal y modelo "de red", más que de tipo vertical y piramidal - la "suavidad" bien expresa la imagen de una normativa que, de alguna manera, se aleja de la obligación impositiva y sancionatoria, presentándose como un tejido de malla ancha que se deja penetrar e integrar por las opciones de los distintos jurídicos. ${ }^{39}$ Se amplía el concepto de regulación respecto a la vinculación, la determinación y la competencia normativa. ${ }^{40}$ Eso no quiere decir que la red no tenga nudos, "núcleos duros", puntos de rigidez, elementos caracterizados por una normativa "fuerte". ${ }^{41}$ La Constitución, junto con los derechos fundamentales, la legislación de distintos sectores de ordenamiento, las normas internacionales y supranacionales aplicadas, las reglas de procedimiento y las sentencias judiciales constituyen las partes resistentes de la estructura jurídica. Estas coexisten con las partes "elásticas", de manera que los sistemas jurídicos tienden a configurarse como conjuntos complejos que presentan, al mismo tiempo, niveles de hard law con fuerte vinculación y niveles de soft law con una flexibilidad y necesidades difíciles de encerrar en esquemas demasiados rígidos. ${ }^{42}$ En contextos jurídicos caracterizados por pluralismo normativo, policentrismo, comunicación osmótica e hibridación entre ordenamientos, la regulación hard y soft se utilizan teniendo en cuenta la particularidad de las situaciones, reflejando una compleja articulación de las teoría de las fuentes..$^{43}$ El derecho se genera a partir de una variedad

36. F. Ost, M. van de Kerchove, Le système juridique entre ordre et désordre, Presses Universitaires de France, París, 1988, pp. 105-111.

37. F. Ost, M. van de Kerchove, De la pyramide au réseau? Pour une théorie dialectique du droit, Publications des Facultés Universitaires Saint-Louis, Bruxelles, 2002, pp. 43 ss., 49 ss.

38. M. R. Ferrarese, Il diritto al presente, pp. 150, 176.

39. Ibíd., pp. 199-200; A. Catania, Metamorfosi del diritto, p. 59.

40. Cfr. C. Scott, "Regolazione gerarchica, pluralismo giuridico e Rule of Law", en Ars Interpretandi, 11, 2006, pp. 115-116. 41. Sobre la coexistencia y complementariedad entre modelo piramidal y modelo reticular, véase M.G. Losano, "Diritto turbolento. Alla ricerca di nuovi paradigmi nei rapporti fra diritti nazionali e normative sovrastatali”, en Rivista internazionale di filosofia del diritto, 82, n. 3, 2005, pp. 425-429.

42. U. Mörth, “Conclusion”, pp. 191-193; L. Senden, Soft Law in European Community Law, pp. 397-398.

43. Di Robilant, “Genealogies of Soft Law”, pp. 504 ss., 545 ss. 
de tipos de reglas, caracterizadas por diferentes grados de "dureza", ${ }^{44}$ configurándose como un mosaico dinámico formado por múltiples piezas, a través de un continuo proceso de interacción entre los distintos elementos que lo componen. ${ }^{45}$

El derecho, como esfera peculiar del discurso normativo, se ha siempre remitido a varias tipologías de normas con el objetivo de influenciar el comportamiento y estas normas han sido diferenciadas según sus distintos grados de intensidad prescriptiva. ${ }^{46}$ En el ámbito de la experiencia jurídica, por lo tanto, la presencia de normas con diferente fuerza de obligación es bastante habitual. El mundo del derecho lo ocupan mandamientos, prohibiciones, órdenes, intimaciones, preceptos, instrucciones, directivas, recomendaciones, consejos, opiniones, avisos, admoniciones, advertencias, propuestas, peticiones, intimaciones, instancias, etc. La distinción entre normas con obligaciones, que por lo tanto tienen una influencia más fuerte y normas sin obligaciones, menos condicionantes, es muy común. Se puede así afirmar que la gradación de niveles de vinculación, que se refiere a la distinción entre soft y hard law, no es un concepto nuevo.

El proceso de formulación de los actos de soft law no define en sí sus características distintivas y salientes. Sin embargo, es oportuno distinguir, según la estructura sintáctica, dos tipos de normas: las incondicionadas y las condicionadas (que a su vez se componen de una prótasis, la parte del enunciado que determina la condición y una apódosis, la parte que determina la consecuencia). Por lo tanto, es posible clasificar las disposiciones de soft law como enunciados que no presentan formulación condicional. De hecho, tal estructura es común a muchas normas que pretenden orientar el comportamiento humano. Los principios, por ejemplo, son normas incondicionadas (sin casos jurídicos) y/o no remiten a ninguna estructura lógica por la cual una consecuencia jurídica se conecta a un caso condicionante (normas con caso jurídico abierto). Además, tienen un lenguaje optativo o evaluativo; indican un objetivo por conseguir; ${ }^{47}$ afirman una razón que dirige hacia una dirección. También, no hay que olvidarse que la mayoría de las veces los actos de soft law enuncian declaraciones de principio. El mismo discurso vale para los standards, como las políticas (policies) que se refieren a bienes colectivos e indican un objetivo por conseguir, respecto a una mejora de algún aspecto - económico político y social - de la vida de la comunidad. ${ }^{48}$

\footnotetext{
44. C. Scott, "Regolazione gerarchica, pluralismo giuridico e Rule of Law", pp. 109 ss.,116-117, 140-141. 45. M. R. Ferrarese, "Mercati e globalizzazione. Gli incerti cammini del diritto", en Politica del diritto, 29, n. 3, 1998 , p. 410. 46. N. Bobbio, Contributi ad un dizionario giuridico, Giappichelli, Torino, 1994, pp. 188-195, 227-230. 47. R. Guastini, Teoria e dogmatica delle fonti, Giuffrè, Milano, 1998, pp. 275, 279, 281-282.

48. R. Dworkin, Taking Rights Seriously, Harvard University Press, Cambridge (Mass.), 1978, pp. 22-23.
} 
También desde el punto de vista de la forma lingüística y de la estructura lógica del enunciado, el soft law no constituye una novedad. En cambio, eso confirma la idea de que los materiales que forman el universo jurídico, y que contribuyen de distintas maneras a determinar los procesos de positivización, son muchos más articulados y complejos con respecto al modelo simplista de la dogmática jurídica. Parece entonces más interesante, desde el punto de vista de la teoría del derecho, dirigirse hacia otras direcciones que permitan captar el valor y la peculiaridad del soft law y de su manera de operar en los actuales contextos jurídicos. Tales direcciones llevan al punto en el cual las fuentes del derecho se cruzan con la interpretación y demuestran como la misma individuación de las fuentes sea el fruto de una actividad interpretativa que se desarrolla dentro de la praxis jurídica.

\section{Fuentes y prácticas interpretativas}

El soft law refleja enteramente esta perspectiva plural que caracteriza cada vez más a los sistemas jurídicos. Está típicamente constituido por textos de distinto origen que registran una variedad de léxicos particulares, con lenguajes específicos y formas expresivas propias, ${ }^{49}$ según los diferentes contextos de la vida social. Tales textos, generalmente producidos a través de esquemas de procedimiento, se consideran en cierto modo jurídicos porque, por su unidad de sentido, forman parte de aquella praxis que definimos como "derecho".

La elasticidad, la flexibilidad y la indeterminación de los contenidos y de los objetivos de los actos del soft law están relacionados tanto con las condiciones de aplicación como con el código de conducta prescrito. Sin duda, el soft law en sus múltiples y diferentes manifestaciones, en calidad de derecho escrito no obligatorio prima facie, prescribe algo aunque de forma atenuada, pero no pueden considerarse como normas que se refieren a casos jurídicos concretos. Los actos del soft law recomiendan un objetivo, indican una disposición que hay que adoptar, un programa al cual atenerse, un aspecto que hay que considerar y un valor que reclama atención. Establecen, en resumen, una orientación que constitutivamente presenta la forma del "más o menos" y requiere una gradualidad en el proceso de realización, influyendo en la producción normativa.

49. F. Viola, G. Zaccaria, Diritto e interpretazione. Lineamenti di teoria ermeneutica del diritto, Laterza, Roma-Bari, 2001, pp. 278-281. 
El soft law, por lo tanto, forma parte del corpus normativo y participa en el proceso de positivización. Es una fuente del derecho, puesto que de alguna forma se le considera capaz de generar reglas jurídicas.

De las "fuentes del derecho" tenemos una noción material y una formal. Según la noción "material", el sintagma se define como cada acto o hecho que produzca derecho. A la luz de esta definición, para saber si un determinado acto o hecho sea una fuente del derecho, es necesario identificar su contenido (si es un acto) o su resultado (si es un hecho). Según la noción "formal”, el sintagma define cada acto o hecho no como efectivo productor de normas, sino como autorizado a producir normas, independientemente de su contenido (si es un acto) o resultado (si es un hecho). De esto se deduce que, para saber si un acto o hecho es fuente del derecho, hay que mirar si en el ordenamiento en cuestión existe alguna norma (en particular una norma sobre la producción jurídica) que autoriza el acto o el hecho a producir derecho. ${ }^{50}$ Ahora bien, a la vista del panorama jurídico actual, que se puede describir como una red en la cual interactúan todas las normas (de distinta proveniencia), se puede afirmar que resulta superada la distinción entre fuente en el sentido material y fuente en el sentido formal (por otra parte útil para objetivos analíticos). Nos encontramos delante de la imposibilidad de reconducir el sistema de las fuentes a enumeraciones esquemáticas, basada por ejemplo en el nomen juris y/o en el procedimiento de formación, es decir en un marco preciso. Existen fuentes que no se pueden colocar exhaustivamente en cualquier nivel de la jerarquía. ${ }^{51}$ Se trata de fuentes extra ordinem, no disciplinadas por disposiciones sobre la producción jurídica sino que operan en base al principio de efectividad (que a su vez ejerce como norma sobre el proceso de producción). ${ }^{52}$ Nuevos actos normativos surgen al lado de los que ya se conocen y consideran. Los intérpretes, junto a la multitud y al constante flujo de las fuentes formales (actos o hechos sujetos a condiciones definidas de validez) e informales (actos o hechos carentes de formas jurídicas normativamente predispuestas y con algún grado de efectividad), ${ }^{53}$ deben identificar el "dato" al cual referir la atribución de significados. Aquí el momento de la elección es relevante y el objeto del proceso hermenéutico se desarrolla en la realidad del ordenamiento con una eficacia diferenciada en cada caso. La fijación del enunciado, a partir del cual se define el criterio

50. R. Guastini, Teoria e dogmatica delle fonti, pp. 57-59, 64-65.

51. F. Modugno, "Fonti del diritto (gerarchia delle)", en Enciclopedia del diritto, Agg. I, Giuffrè, Milano, 1997, pp. 585-586. 52. A. Pizzorusso, "Delle fonti del diritto", en Commentario del codice civile, en A. Scialoja e G. Branca (eds.), Zanichelli, Bologna - Soc. Ed. del Foro Italiano, Roma, 1977, p. 541.

53. L. Ferrajoli, Principia iuris. Teoria del diritto e della democrazia. 1. Teoria del diritto, Laterza, Roma-Bari, 2007 pp. 418 , 921-922. 
efectivo de regulación, implica la necesidad de definir previamente la fuente a la cual se refiere. A la tradicional tarea de inferir la norma de su fuente, se añade la de la búsqueda de la misma fuente que hay que reconstruir. Tal proceso de determinación no siempre resulta rigurosamente unívoco y capaz de prescindir de la búsqueda del objetivo que se quiere perseguir. Eso requiere que la interpretación de la fuente sea vinculada al marco de las relaciones sociales dentro el cual la fuente puede reconocerse como tal. La configuración de las fuentes sufre un proceso de revisión hacia un ordenamiento "abierto" a las practicas sociales de determinación del derecho. Nos encontramos delante a una disociación entre el conjunto de las fuentes formales y el que viene aceptado por la comunidad jurídica (más amplio y comprensivo de la fuentes informales). ${ }^{54}$

"Fuente" es un término que remite al conjunto de factores que influyen en la producción de las normas. Esta influencia puede variar: hay fuentes que proporcionan al operador jurídico una norma inmediatamente válida y fuentes que ofrecen solo ideas, inspiraciones y orientaciones a partir de las cuales los operadores tienen que inferir las normas. ${ }^{55}$ Así, las fuentes presentan diferentes grados de objetivación, pero todas se presentan como recursos oficiales para inferir las reglas para casos particulares. $\mathrm{Al}$ mismo tiempo, estas son fruto de la interpretación y la decisión y operan como fundamentos para las soluciones jurídicas. ${ }^{56}$ Bajo esta perspectiva, los resultados jurídicos derivan no solo de normas obtenidas a través de procedimientos formalmente válidos, sino también de normas no obtenidas, ni obtenibles, a través de tales procedimientos. ${ }^{57}$

El uso de fuentes remite por lo tanto al momento interpretativo y aplicativo, es decir, que la búsqueda de las fuentes se convierte en una cuestión de interpretación y de aplicación del derecho. ${ }^{58}$ A raíz de las normas existen diferentes materiales jurídicos, heterogéneos y con distinta fuerza vinculante, que forman un conjunto normativo en potencia que hay que actualizar. El derecho positivo se manifiesta bajo este punto de vista como una acción solidaria de sujetos, institucionales y no institucionales, que con su actividad hermenéutica identifican y articulan el discurso de las fuentes, dentro de una praxis en la cual la normativa jurídica es el resultado de factores complejos que se conectan e interactúan.

54. P. G. Monateri, "Fonti del diritto", in Digesto delle Discipline Privatistiche, sez. civ., VIII, Utet, Torino, 1992, p. 392; A. Pizzorusso, "Delle fonti del diritto", p. 543.

55. A. Ross, On Law and Justice, Stevens \& Sons Ltd., London, 1958, p. 77.

56. G. Zaccaria, La comprensione del diritto, p. 46.

57. F. Schauer, Playing by the Rules. A Philosophical Examination of Rule-Based Decision-Making in Law and in Life, Clarendon Press, Oxford, 1991, p. 201.

58. F. Viola, G. Zaccaria, Diritto e interpretazione, pp. 316-317, 326, 330; B. Pastore, "Le fonti e la rete: il principio di legalità rivisitato", en G. Brunelli, A. Pugiotto, P. Veronesi (eds.), Scritti in onore di Lorenza Carlassare. Il diritto costituzionale come regola e limite al potere. I. Delle fonti del diritto, Jovene, Napoli, 2009, pp. 269-272. 
La positivización jurídica se cumple a partir de lugares de autoridad, pero ninguno de ellos es autosuficiente o conclusivo, prescindiendo de la actividad interpretativa, y solo se manifiesta en la practica jurídica. ${ }^{59}$ El proceso de soft law confirma que la interpretación representa un aspecto interior en las dinámicas de las fuentes. $\mathrm{Al}$ mismo tiempo, este proceso demuestra de manera paradigmática que la teoría de las fuentes no se puede concebir bajo la perspectiva de la reconstrucción estática entre los actos y las normas que proceden de ellos (como "mónadas" cerradas en sí mismas, consideradas de manera atomística y netamente distinguibles), ${ }^{60}$ sino al contrario se puede afirmar que conecta de manera constitutiva con la teoría de la interpretación.

El derecho se manifiesta como un tejido, una red de interrelaciones, dentro de las cuales unas reglas ligeras, fluidas y elásticas, coexisten con disciplinas plenamente obligatorias, desempeñando distintos roles y respondiendo a la exigencia de flexibilidad y adaptabilidad de contextos sociales cada vez más complejos. La normativa resulta, en este marco, no monolítica sino caracterizada por un considerable nivel de gradualidad. Esta se pone en un continuum de distintas posibles relaciones respecto a la vigencia y validez de los materiales jurídicos, su efectividad, el rol que estos desempeñan en el proceso de positivización, su obligatoriedad, junto con su aspecto coercitivo y nivel de observancia. Los grados de normatividad son diferentes tanto en el peso como en el valor y por lo tanto eso remite al uso, aplicabilidad, aceptación y observancia de las reglas jurídicas.

Todo este proceso está conectado con una continua y extensa praxis hermenéutica, articulada en distintos niveles, tanto "técnicos" y reconocidos en el ordenamiento, como aquellos no institucionalizados dentro de los cuales actúan los sujetos que experimentan la validez del derecho y lo eligen como razón para tomar decisiones. De esta manera, los materiales jurídicos presentan distintos niveles de elaboración y determinación. La positividad se gradúa. ${ }^{61}$ El derecho puede ser más o menos positivo y consta de factores múltiples, distribuidos en los distintos niveles de su producción, integración, reconstrucción y aplicación.

En la fase actual de profunda reestructuración del paisaje jurídico, siendo perjudicada la unidad y la rigidez del sistema de las fuentes, el ordenamiento existe en la relación que conecta múltiples actos normativos, diversamente vinculantes, con las actividades interpretativas que estos desarrollan. La efectividad, por lo tanto, se presenta, no solo

59. F. Viola, G. Zaccaria, Diritto e interpretazione, p. 331.

60. A. Ruggeri, "Prospettive metodiche di ricostruzione del sistema delle fonti e Carte internazionali dei diritti, tra teoria delle fonti e teoria dell'interpretazione", en Ragion pratica, 18, 2002, p. 73.

61. L. Gianformaggio, Filosofia e critica del diritto, Giappichelli, Torino, 1995, p. 31. 
como respuesta de conformidad y obediencia de los destinatarios a las normas, sino también como respuesta de reconocimiento de los asociados. ${ }^{62}$ Estos, a partir de varios elementos (infraestatales, estatales, supranacionales, internacionales) construyen hermenéuticamente un ordenamiento jurídico que presenta un intrínseco carácter plural, polivalente y dinámico.

Soft law y hard law coexisten como áreas normativas a menudo interconectadas en el proceso de articulación progresiva del material jurídico. Ambos contribuyen al proceso de orientación de los comportamientos y a la toma de decisiones, en relación a los efectivos ámbitos sociales y especificidades regulativas. Tal proceso caracteriza el derecho como una actividad ${ }^{63}$ que presenta constitutivamente una gradualidad normativa más o menos intensa. 